1. Kalluri, R., and Sukhatme, V.P. 2000. Fibrosis and angiogenesis. Curr. Opin. Nephrol. Hypertens. 9:413-418.

2. Belperio, J., et al. 2005. Role of CXCR2/CXCR2 ligands in vascular remodeling during bronchiolitis obliterans syndrome. J. Clin. Invest. 115:1150-1162. doi:10.1172/JCI200524233.

3. Estenne, M., and Hertz, M.I. 2002. Bronchiolitis obliterans after human lung transplantation. Am J. Respir. Crit. Care Med. 166:440-444.

4. Turner-Warwick, M. 1963. Precapillary systemicpulmonary anastomoses. Thorax. 18:225-237.

5. Belperio, J.A., et al. 2001. Critical role for the chemokine MCP-1/CCR2 in the pathogenesis of bronchiolitis obliterans syndrome. J. Clin. Invest. 108:547-556. doi:10.1172/JCI200112214.

6. Belperio, J.A., et al. 2000. CXC chemokines in angiogenesis. J. Leukoc. Biol. 68:1-8.

7. Fernandez, F.G., et al. 2004. Airway epithelium is the primary target of allograft rejection in murine obliterative airway disease. Am. J. Transplant. 4:319-325.

8. Murakawa, T., et al. 2005. Simultaneous LFA-1 and CD40 ligand antagonism prevents airway remodeling in orthotopic airway transplantation: implications for the role of respiratory epithelium as a modulator of fibrosis. J. Immunol. 174:3869-3879.

9. Minamoto, K., and Pinsky, D.J. 2002. Recipient iNOS but not eNOS deficiency reduces luminal narrowing in tracheal allografts. J. Exp. Med. 196:1321-1333.

10. Neuringer, I.P., et al. 2002. Epithelial kinetics in mouse heterotopic tracheal allografts. Am. J. Transplant. 2:410-419.

11. Reader, J.R., et al. 2003. Pathogenesis of mucous cell metaplasia in a murine asthma model. Am. J. Pathol. 162:2069-2078.

12. Kasper, M., and Haroske, G. 1996. Alterations in the alveolar epithelium after injury leading to pulmonary fibrosis. Histol. Histopathol. 11:463-483.

13. Howat, W.J., Holgate, S.T., and Lackie, P.M. 2002. TGF-beta isoform release and activation during in vitro bronchial epithelial wound repair. Am.J. Physiol. Lung Cell Mol. Physiol. 282:L115-L123.

14. Morishima, Y., et al. 2001. Triggering the induction of myofibroblast and fibrogenesis by airway epithelial shedding. Am. J. Respir. Cell Mol. Biol. 24:1-11.

15. Zhang, S., Smartt, H., Holgate, S.T., and Roche, W.R. 1999. Growth factors secreted by bronchial epithelial cells control myofibroblast proliferation: an in vitro co-culture model of airway remodeling in asthma. Lab. Invest. 79:395-405.

16. Phillips, R.J., et al. 2004. Circulating fibrocytes traffic to the lungs in response to CXCL12 and mediate fibrosis. J. Clin. Invest. 114:438-446. doi:10.1172/ JCI200420997.

17. Palmer, S.M., et al. 2000. Gastroesophageal reflux as a reversible cause of allograft dysfunction after lung transplantation. Chest. 118:1214-1217.

18. Chakinala, M.M., and Walter, M.J. 2004. Community acquired respiratory viral infections after lung transplantation: clinical features and longterm consequences. Semin. Thorac. Cardiovasc. Surg. 16:342-349.

19. Zamora, M.R. 2004. Cytomegalovirus and lung transplantation. Am. J. Transplant. 4:1219-1226.

20. Penfold, M.E., et al. 1999. Cytomegalovirus encodes a potent alpha chemokine. Proc. Natl. Acad. Sci.U. S. A. 96:9839-9844.

21. Knoop, C., Haverich, A., and Fischer, S. 2004. Immunosuppressive therapy after human lung transplantation. Eur. Respir. J. 23:159-171.

22. Selman, M., and Pardo, A. 2002. Idiopathic pulmonary fibrosis: an epithelial/fibroblastic cross-talk disorder. Respir. Res. [serial online]. 3:3. http://respiratory-research.com/content $/ 3 / 1 / 3$.

\title{
The role of hepatic insulin receptors in the regulation of glucose production
}

\author{
Alan D. Cherrington
}

Department of Molecular Physiology and Biophysics, Vanderbilt University School of Medicine, Nashville, Tennessee, USA.

\begin{abstract}
The inability of insulin to suppress hepatic glucose production (HGP) is a key defect found in type 2 diabetes. Insulin inhibits HGP through both direct and indirect means, the latter of which include inhibition of glucagon secretion, reduction in plasma nonesterified fatty acid level, decrease in the load of gluconeogenic substrates reaching the liver, and change in neural signaling to the liver. Two studies in this issue of the JCI demonstrate that selective changes in the expression of insulin receptors in mouse liver do not have a detectable effect on the ability of insulin to inhibit HGP (see the related articles beginning on pages 1306 and 1314). These provocative data suggest that the indirect effects of insulin on the liver are the primary determinant of HGP in mice.
\end{abstract}

\section{Introduction}

Until late 1987, it was believed that insulin's ability to reduce hepatic glucose production (HGP) resulted from the direct interaction of the hormone with its receptor in the hepatocyte plasma membrane. This belief was called into question when Prager et al. (1) noted that in obese, nondiabetic humans, suppression of glucose production could occur in response to insulin

Nonstandard abbreviations used: HGP, hepatic glucose production; LIRKO, liver insulin receptor knockout; NEFA: nonesterified fatty acid; NHGO, net hepatic glucose output.

Conflict of interest: The author has declared that no conflict of interest exists.

Citation for this article: J. Clin. Invest. 115:1136-1139 (2005). doi:10.1172/JCI200525152 infusion, even when the estimated portal vein insulin concentration did not rise. These results suggested that insulin also reduces hepatic glucose output by indirect mechanisms. Subsequent work by others supported this concept (2-5), and it is now recognized that insulin can inhibit HGP by both direct and indirect means (Figure 1).

Indirect actions of insulin on the liver The indirect actions of insulin on HGP are diverse. Glucagon secretion from the $\alpha$ cell of the pancreas is diminished by insulin, which in turn causes a reduction in HGP (6). Likewise, nonesterified fatty acid (NEFA) release from the adipocyte is reduced by insulin, and a reduction in the supply of NEFAs to the liver causes an increase in hepatic glycolytic flux, resulting in glucose-6-phosphate exiting the liver after being converted to lactate rather than glucose (3). Additionally, the effect of insulin on fat and muscle reduces the supply of gluconeogenic precursors reaching the liver, again reducing $\operatorname{HGP}(2,7)$. More recently, insulin's action in the brain has been postulated to play a role in the regulation of HGP (8). It is known that the brain can sense the circulating insulin level (9) and that it provides neural input to the liver (10). Further, it has been shown that infusion of insulin into the third ventricle of rats can reduce glucose production (8). Likewise, blocking insulin action in the rat hypothalamus impairs the ability of a physiologic rise in circulating insulin to inhibit HGP (8).

\section{Direct actions of insulin on the liver}

The exploration of insulin's indirect effects on the liver called into question the physiologic relevance of the hormone's direct hepatic effect, even though numerous in vitro studies had shown it to exist. Perhaps the best in vivo demonstration of the hormone's direct effect on the liver comes from studies conducted in the conscious overnight-fasted dog, in which changes in plasma insulin were brought about selectively in the liver using the pancreatic clamp technique. 
In the absence of any change in arterial plasma glucagon or insulin levels, plasma NEFA level, gluconeogenic precursor load reaching the liver, or insulinization of the brain, hepatic glucose output rose 3-fold when portal vein insulin fell by $75 \%$, and decreased by $50 \%$ when portal vein insulin rose by $75 \%(7,2)$. These data leave no doubt that the liver responds directly, rapidly, and sensitively to the insulin in the plasma perfusing it.

The relative importance of the direct and indirect effects of insulin in regulating HGP was also examined in the overnightfasted conscious dog (11). Somatostatin was infused to inhibit endogenous insulin and glucagon release, and both hormones were replaced intraportally at basal rates. After a 40-minute control period, the route of insulin delivery was switched from the portal vein to a femoral vein. As a result, the arterial insulin level was doubled, and the portal vein insulin level was halved. This led to a rise in HGP and hyperglycemia (11), which proves the dominance of insulin's direct effects on the liver in this species.

\section{Manipulation of hepatic insulin receptors in mice}

The importance of the direct interaction of insulin with its hepatic receptors is supported by Fisher et al.'s observations that hepatic insulin resistance and markedly elevated plasma insulin levels were present in liver insulin receptor knockout (LIRKO) mice (12). These authors concluded that insulin's direct and indirect effects both require the presence of hepatic insulin receptors. The overall health of the LIRKO animals has been questioned, however (13), and the long-term absence of hepatic insulin receptors may give rise to a unique phenotype.

In light of the findings of Fisher et al. (12), the results of Buettner et al. (13) and Okamoto et al. (14) described in this issue of the JCI are surprising. Buettner et al. showed that acute inhibition of insulin receptor expression in mice by the injection of an antisense oligonucleotide did not inhibit insulin's ability to suppress glucose production (13). In a companion paper from the same lab, Okamoto et al. showed that restoration of insulin receptors in the livers of mice otherwise lacking the insulin receptor did not restore insulin's ability to reduce glucose production in vivo (14).

\section{Figure 1}

Mechanisms by which insulin can inhibit HGP in vivo.

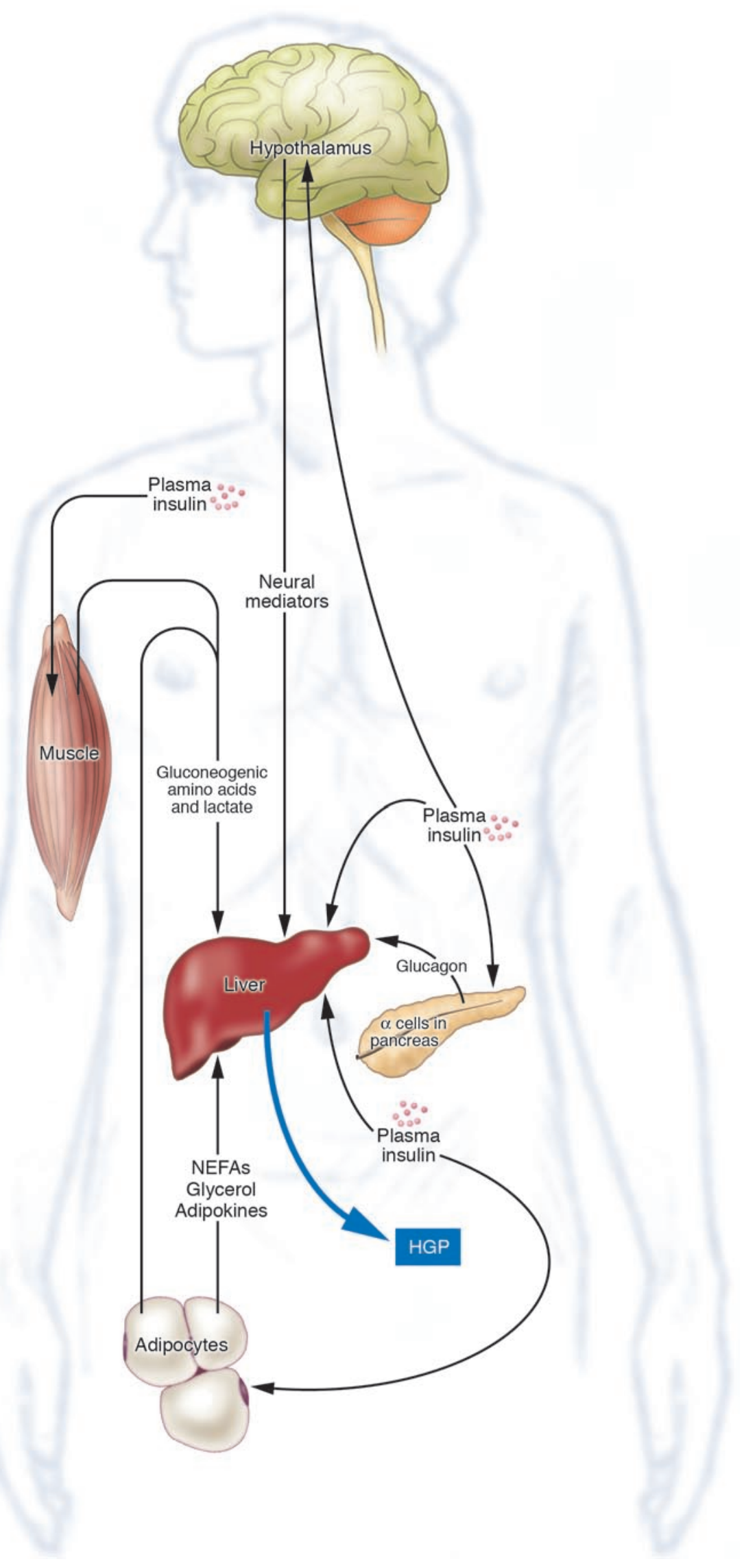


Taken at face value, these results suggest that the role of hepatic insulin receptors in the control of HGP in mice is limited and that the indirect mechanisms of insulinmediated HGP inhibition are dominant.

\section{Methodological considerations}

The measurement of endogenous glucose production during an insulin clamp in the mouse is difficult. One must therefore consider the possibility that changes in the direct effect of insulin on endogenous glucose production occurred in the 2 aforementioned studies $(13,14)$ but could not be detected. To assess the responsiveness of HGP during a euglycemic hyperinsulinemic clamp, one must subtract the glucose infusion rate from the rate of glucose turnover. Given the magnitude of these rates in mice as reported by the authors $(54 \pm 5$ to $40 \pm 4 \mathrm{mg} / \mathrm{kg}$ per minute), the differences are likely to have high variance. The incremental change in the plasma insulin level brought about by Buettner et al. caused a 50\% fall in HGP (i.e., a difference of about $15 \mathrm{mg} / \mathrm{kg}$ per minute) in control animals (13). If two-thirds of this fall was attributable to insulin's direct effect on the liver, then if that effect was reduced by $50 \%$ as a result of the near-absence of hepatic insulin receptors, one would expect a decline of $10 \mathrm{mg} / \mathrm{kg}$ per minute. In that case, HGP during the clamp period in the 2 groups would differ by only $5 \mathrm{mg} / \mathrm{kg}$ per minute. Given the variance in the calculated HGP, the small magnitude of the difference in HGP in the 2 groups, and the fact that the basal HGP was not determined in the clamp experiments, the study probably did not have the power to detect a reduction of even $50 \%$ in the direct effect of insulin on the liver. As a result, although a direct effect of insulin on HGP was not evident (13), it cannot be ruled out on the basis of the data presented. However, the error is less relevant to the findings of the companion paper from Okamoto et al., as in that case there should have been a full direct effect (i.e., a decrement of $10 \mathrm{mg} / \mathrm{kg}$ per minute in glucose production) in the mice with restored insulin receptors, and this did not appear to occur (14). It must be remembered, however, that a modest decrease might well have gone undetected for the methodological reasons discussed above. Nevertheless, the combined papers make a compelling case for the role of indirect mechanisms in the control of HGP in mice.

As a small amount (approximately 5\%) of hepatic insulin receptors may have been present even 1 week after treatment with the antisense oligonucleotide in the Buettner et al. study (13), it is possible that there were still enough receptors present to allow insulin to reduce HGP to some extent. But it becomes harder to explain why $5 \%$ of residual receptors would be enough to suppress HGP in this model when $60 \%$ of receptors in the study by Okamoto et al. failed to mediate insulin's effect. Another surprising finding in the Buettner et al. paper is the observation that insulin clearance did not change despite the change in hepatic insulin receptor expression. Given the importance of liver insulin receptors in insulin degradation $(15,16)$, one would have expected to see a greater increase in plasma insulin levels in the absence of hepatic insulin receptors. Interestingly, in the Okamoto et al. study, the same increase in plasma insulin was observed in response to insulin infusion in wild-type mice and mice with insulin receptors in the liver but not elsewhere in the body (14). An additional confounder in this study is the fall in adiponectin levels that was observed during insulin infusion (14). The role of adiponectin in determining the liver's sensitivity to insulin is one we are striving to better understand, and these animal models may be useful in that respect.

\section{Physiological considerations}

The authors' conclusion that changes in the expression of insulin receptors in the liver do not affect the ability of insulin to inhibit HGP prompts the question: How did the fall in HGP reported by Buettner et al. (13) come about? The data from this study show that the observed inhibition of HGP cannot be attributed to a fall in plasma glucagon level, plasma NEFA level, or gluconeogenic precursor load reaching the liver. The only indirect mechanism that appears viable is that of an action of insulin on the brain. If this is the mechanism by which the inhibition of HGP came about, then 3 questions arise: First, do mice have constitutively high neural drive to the liver? Second, does the role of the brain in controlling HGP become more important when hepatic insulin receptors are absent? Third, is the regulation of HGP in mice unique, or do these findings also apply to other species? The increased ability to manipulate the mouse genome by way of conditional mutagenesis should allow the authors to address some of these remaining questions in due course.

With regard to the first question, it must be remembered that basal glucose produc- tion in mice is $10-15$ times greater (per kg of body weight) than in humans and dogs, while basal plasma glucagon levels are similar in all 3 species. It is therefore possible that, in mice, the liver does have substantial neural input in the basal state. It is also possible that removal of hepatic insulin receptors actually leads to increased neural control of glucose production as a protective response. In either case, caution must be used in extrapolating these findings to non-rodent mammals.

Even though the use of the antisense oligonucleotide targeted against the insulin receptor obviated the need for long-term absence of the hepatic insulin receptor, it still created an ever-increasing deficiency of insulin receptors progressively over the course of 1 week, possibly allowing the expression of compensatory mechanisms. This is a very different situation from one in which there is an acute reduction in portal vein insulin levels (over the course of minutes, as in the previously described dog studies; ref. 2) or in the number of hepatic insulin receptors. Acute reduction of either exposes the physiologic role of those receptors (or the direct effect of insulin on the liver), while a more prolonged deficiency examines a combination of their physiologic roles and the adaptive response of the animal to their absence.

\section{Summary}

It is clear, based on all of the aforementioned studies, that insulin's control of HGP is not only important but much more complex than previously imagined. In this respect, these 2 studies contribute exciting, if somewhat provocative, new insight into this area. Data from studies of dogs clearly point to the dominance of insulin's direct effects on the liver, while data from studies of mice suggest that it is insulin's indirect effects that are dominant. It is known that in humans, insulin inhibits glucose production by both direct and indirect means $(17,18)$, but it remains to be seen whether the lessons learned from these animal models can be applied to the treatment of patients with diabetes.

Address correspondence to: Alan D. Cherrington, Department of Molecular Physiology and Biophysics, Vanderbilt University School of Medicine, 704 Robinson Research Building, Nashville, Tennessee 37232-0615, USA. Phone: (615) 3227013; Fax: (615) 343-0490; E-mail: alan. cherrington@vanderbilt.edu. 
1. Prager, R., Wallace, P., and Olefsky, J.M. 1987. Direct and indirect effects of insulin to inhibit hepatic glucose output in obese subjects. Diabetes. 36:607-611.

2. Sindelar, D.K., Balcom, J.H., Chu, C.A., Neal, D.W., and Cherrington, A.D. 1996. A comparison of the effects of selective increases in peripheral or portal insulin on hepatic glucose production in the conscious dog. Diabetes. 45:1594-1604.

3. Sindelar, D.K., et al. 1997 . The role of fatty acids in mediating the effects of peripheral insulin on hepatic glucose production in the conscious dog. Diabetes. 46:187-196.

4. Ader, M., and Bergman, R.N. 1990. Peripheral effects of insulin dominate suppression of fasting hyperglycemia. Am. J. Physiol. 258:E1029-E1032.

5. Giacca, A., et al. 1992. Importance of peripheral insulin levels for insulin-induced suppression of glucose production in depancreatized dogs. J. Clin. Invest. 90:1769-1777

6. Ito, K., et al. 1995. Exogenous insulin dose-dependently suppresses glucopenia-induced glucagon secretion from perfused rat pancreas. Metabolism. 44:358-362.
7. Sindelar, D.K., et al. 1998. Basal hepatic glucose production is regulated by the portal vein insulin concentration. Diabetes. 47:523-529.

8. Obici, S., Zhang, B.B., Karkanias, G., and Rossetti, L. 2002. Hypothalamic insulin signaling is required for inhibition of glucose production. Nat. Med. 8:1376-1382.

9. Davis, S.N., et al. 1995. Evidence that the brain of the conscious dog is insulin sensitive. J. Clin. Invest. 95:593-602.

10. la Fleur, S.E., Kalsbeek, A., Wortel, J., and Buijs, R.M. 2000. Polysynaptic neural pathways between the hypothalamus, including the suprachiasmatic nucleus, and the liver. Brain Res. 871:50-56.

11. Cherrington, A.D., et al. 2002. Metabolic importance of portal insulin delivery. Diabetes. 51(Suppl. 2):A313.

12. Fisher, S.J., and Kahn, C.R. 2003. Insulin signaling is required for insulin's direct and indirect action on hepatic glucose production. J. Clin. Invest. 111:463-468. doi:10.1172/JCI200316426.

13. Buettner, C., et al. 2005. Severe impairment in liver insulin signaling fails to alter hepatic insulin action in conscious mice. J. Clin. Invest. 115:1306-1313. doi:10.1172/JCI200523109.

14. Okamoto, H., Obici, S., Accili, D., and Rossetti, L. 2005. Restoration of liver insulin signaling in Insr knockout mice fails to normalize hepatic insulin action. J. Clin. Invest. 115:1314-1322. doi:10.1172/ JCI200523096.

15. Duckworth, W.C., Bennett, R.G., and Hamel, F.G. 1997. The significance of intracellular insulin to insulin action. J. Invest. Med. 45:20-27.

16. Duckworth, W.C., Bennett, R.G., and Hamel, F.G. 1998. Insulin acts intracellularly on proteasomes through insulin-degrading enzyme. Biochem. Biophys. Res. Comm. 244:390-394.

17. Lewis, G.F., Zinman, B., Groenewoud, Y., Vranic, M., and Giacca, A. 1996. Hepatic glucose production is regulated both by direct hepatic and extrahepatic effects of insulin in humans. Diabetes. 45:454-462.

18. Cherrington, A.D., Edgerton, D., and Sindelar, D.K. 1998. The direct and indirect effects of insulin on hepatic glucose production. Diabetologia. 41:987-996.

\title{
Contribution of adipose tissue and de novo lipogenesis to nonalcoholic fatty liver disease
}

\author{
Shinji Tamura and lichiro Shimomura
}

Department of Internal Medicine and Molecular Science, Graduate School of Medicine, Osaka University, Suita, Osaka, Japan.

\begin{abstract}
Nonalcoholic fatty liver disease (NAFLD) is a component of the metabolic syndrome, with a clinical spectrum ranging from simple fatty liver to steatohepatitis, cirrhosis, and hepatocellular carcinoma. The primary event of NAFLD is the accumulation of triacylglycerols (TAGs) in hepatocytes. In this issue of the JCI, Donnelly et al. report on their use of stable isotope methodology to show that fatty acids stored in adipose tissue and fatty acids newly made within the liver through de novo lipogenesis are the major sources of TAGs in the liver and are secreted as lipoproteins in NAFLD (see the related article beginning on page 1343).
\end{abstract}

Nonalcoholic fatty liver disease (NAFLD) is the most common cause of abnormal results in liver-function tests. This clinical situation is really a continuum of diseases that includes simple (benign) fatty liver, nonalcoholic steatohepatitis (NASH), cirrhosis, and hepatocellular carcinoma (1). NAFLD is defined as liver steatosis in patients who do not consume enough alcohol to cause hepatic injury. Although some drugs or genetic abnormalities can cause NAFLD, the majority of cases are associated with obesity, insulin resistance,

Nonstandard abbreviations used: ChREBP, carbohydrate responsive element-binding protein; DNL, de novo lipogenesis; NAFLD, nonalcoholic fatty liver disease; NASH, nonalcoholic steatohepatitis; NEFA, nonesterified fatty acid; TAG, triacylglycerol.

Conflict of interest: The authors have declared that no conflict of interest exists.

Citation for this article: J. Clin. Invest. 115:1139-1142 (2005). doi:10.1172/JCI200524930. and type 2 diabetes. In order to clarify the pathogenesis of this disease and identify potential therapeutic targets, an increased understanding of the dynamics of triacylglycerol (TAG) metabolism in the liver in relation to whole-body metabolic status is needed. Sources of hepatic TAGs include dietary TAGs that are transported via chylomicrons from the intestine to adipose tissue or to the liver, where they are secreted via lipoproteins, in addition to TAGs that are synthesized from fatty acids and glycerol in the liver. Fatty acids required for TAG synthesis are available from both the plasma nonesterified fatty acid (NEFA) pool and the pool of fatty acids newly synthesized within the liver through de novo lipogenesis (DNL). TAGs present in the liver may be stored as lipid droplets or secreted into the blood as VLDLs; they may also enter the oxidation pathway (Figure 1). The study by Don- nelly et al. in this issue of the JCI examines hepatic lipoprotein metabolism in humans and describes the major sources of hepatic and plasma lipoprotein TAG in NAFLD (2).

\section{The NEFA pool}

Donnelly et al. found that the plasma NEFA pool accounts for approximately $60 \%$ of TAG content in the livers of NAFLD patients, which reflects the importance of the NEFA pool in the pathogenesis of NAFLD (2). The hepatic uptake of fatty acids is not regulated and, as a result, plasma fatty acid concentration is directly related to the influx of fatty acids to the liver. The authors report that, in the fasted state, adipose tissue contributes approximately $80 \%$ of fatty acid content to the plasma NEFA pool, and even in the fed state, this contribution remains at approximately $60 \%$. Thus, the overproduction of fatty acids in adipose tissues that flow to the liver via the NEFA pool is the most likely explanation for excess TAG accumulation in NAFLD. In insulin-resistant states, insulin does not fully suppress the activity of hormone-sensitive lipase, which catalyzes the hydrolytic release of fatty acids from the TAG moiety and results in enhanced lipolysis and flux of fatty acids to the plasma NEFA pool (Figure 1). In addi- 